\title{
Computing the number of $h$-edge spanning forests in complete bipartite graphs
}

\author{
Rebecca J. Stones $\|^{*}$ \\ School of Mathematical Sciences, Monash University, Australia \\ Department of Mathematics and Statistics, Dalhousie University, Canada \\ received $6^{\text {th }}$ Oct. 2012, revised $28^{\text {th }} \mathrm{Feb} .2014$, accepted $25^{\text {th }}$ Apr. 2014.
}

Let $f_{m, n, h}$ be the number of spanning forests with $h$ edges in the complete bipartite graph $K_{m, n}$. Kirchhoff's Matrix

Tree Theorem implies $f_{m, n, m+n-1}=m^{n-1} n^{m-1}$ when $m \geq 1$ and $n \geq 1$, since $f_{m, n, m+n-1}$ is the number of spanning trees in $K_{m, n}$. In this paper, we give an algorithm for computing $f_{m, n, h}$ for general $m, n, h$. We implement this algorithm and use it to compute all non-zero $f_{m, n, h}$ when $m \leq 50$ and $n \leq 50$ in under 2 days.

MSC 2010: 68R10, 05C85, 05C30, 05C38, $05 \mathrm{C} 05$

Keywords: complete bipartite graph, spanning forest

\section{Introduction}

A consequence of Kirchhoff's Matrix Tree Theorem is that the number of spanning trees in the complete bipartite graph $K_{m, n}$ is $m^{n-1} n^{m-1}$ when $m \geq 1$ and $n \geq 1$. In this paper, we consider a generalisation of this counting problem: the number $f_{m, n, h}$ of $h$-edge spanning forests in $K_{m, n}$. The number of spanning trees in $K_{m, n}$ is $f_{m, n, m+n-1}$ when $m \geq 1$ and $n \geq 1$, since spanning trees of $K_{m, n}$ have $m+n-1$ edges.

The numbers $f_{m, n, h}$ arise in relation to the Tutte polynomial of $K_{m, n}$. For a general graph $G=(V, E)$, the Tutte polynomial is

$$
T_{G}(x, y)=\sum_{A \subseteq E}(x-1)^{k(A)-k(E)}(y-1)^{k(A)+|A|-|V|}
$$

where $k(A)$ is the number of connected components in the graph $(V, A)$. Importantly, for the Tutte polynomial, we take $0^{0}=1$. Thus, the number of spanning forests of $G$ is given by

$$
\begin{aligned}
T_{G}(2,1) & =\sum_{A \subseteq E} 0^{k(A)+|A|-|V|} \\
& =\#\{A \subseteq E: k(A)+|A|=|V|\} \\
& =\text { number of spanning forests of } G,
\end{aligned}
$$

*Email: rebecca.stones82@gmail. com. Stones supported by NSFC grant 61170301 . Stones would also like to thank AARMS for their support.

1365-8050 @ 2014 Discrete Mathematics and Theoretical Computer Science (DMTCS), Nancy, France 
since $k(A)+|A|=|V|$ if and only if $A$ is a spanning forest.

In the present paper, we are interested in the case of when $G=K_{m, n}$, and will look for $h$-edge spanning forests. In this situation,

$$
T_{K_{m, n}}(2,1)=\sum_{h \geq 0} f_{m, n, h} .
$$

For general graphs $G=(V, E)$, Myrvold [10] gave an algorithm for computing the number of $k$ component spanning forests of $G$. Bjöklund et al. [4] described an algorithm that could compute $T_{G}(2,1)$ in time $2^{|V|}|V|^{O(1)}$ by using Kirchhoff's Matrix Tree Theorem for each of the $2^{|V|}$ subsets of $V$, then combining the results together with Inclusion-Exclusion. Other relevant work for the enumeration of spanning forests includes [9]. Porter [11] gave an algorithm for generating the spanning trees of $K_{m, n}$. Farr and McDiarmid [5] showed that computing the number of circuits in $G$ is \#P-complete ${ }^{(i)}$

Bounds for the number of spanning forests in graphs were given by Teranishi [13], from which we can deduce

$$
T_{K_{m, n}}(2,1) \geq \sum_{k \geq 0}\left(\frac{\min (m, n)}{2}\right)^{m+n-k}\left(\begin{array}{c}
m+n \\
k
\end{array}\right)
$$

for all $m \geq 0$ and $n \geq 0$. Jin and Liu [8] gave a simple formula for the number of rooted spanning forests in $K_{m, n}$ (see also [7] and [12]).

This paper instead heads in a different direction to previous work: we derive algorithms for enumerating $h$-edge spanning forests in $K_{m, n}$.

\section{Basic results}

To be clear, all forests in this paper will be labelled. Let $\mathbb{N}=\{0,1,2, \ldots\}$. The following lemma gives the boundary conditions on $f_{m, n, h}$.

Lemma 1 Suppose $m, n, h \in \mathbb{N}$. Then

- $f_{m, n, 0}=1$,

- if $h \geq 1$, then $f_{0, n, h}=f_{m, 0, h}=0$,

- if $m \geq 1$ and $n \geq 1$, then $f_{m, n, h}>0$ if and only if $0 \leq h \leq m+n-1$,

- if $m \geq 1$ and $n \geq 1$, then $f_{m, n, m+n-1}=m^{n-1} n^{m-1}$.

Proof: If $h=0$, then there is exactly one subgraph of $K_{m, n}$ with no edges (and it is a spanning forest), so $f_{m, n, 0}=1$. If $m=0$ or $n=0$, then $K_{m, n}$ has no edges, and thus we obtain the second bulleted item.

Now assume $m \geq 1$ and $n \geq 1$. Since $K_{m, n}$ is connected, it has a spanning tree, which must have exactly $m+n-1$ edges. By deleting edges from this spanning tree, we find $h$-edge forests in $K_{m, n}$ for all $0 \leq h \leq m+n-1$. Hence $f_{m, n, h}>0$ when $0 \leq h \leq m+n-1$.

Now assume $A$ is an $h$-subset of the edges in $K_{m, n}$, where $m \geq 1$ and $n \geq 1$ and $h \geq m+n$. To be a spanning forest, we need $k(A)+h=m+n$ as per (1), but if $h \geq m+n$, then $k(A) \leq 0$, giving a contradiction.

(i) \#P is the set of counting problems which ask for the number of "yes" instances for decision problems in NP. A \#P problem is in \#P-complete whenever any other problem in \#P can be reduced to it by a polynomial-time counting reduction. 


\begin{tabular}{|c|c|c|c|c|c|c|c|c|c|c|c|c|c|}
\hline$m$ & $n$ & $h=0$ & 1 & 2 & 3 & 4 & 5 & 6 & 7 & 8 & 9 & 10 & 11 \\
\hline 1 & 1 & 1 & 1 & & & & & & & & & & \\
\hline 1 & 2 & 1 & 2 & 1 & & & & & & & & & \\
\hline 1 & 3 & 1 & 3 & 3 & 1 & & & & & & & & \\
\hline 1 & 4 & 1 & 4 & 6 & 4 & 1 & & & & & & & \\
\hline 1 & 5 & 1 & 5 & 10 & 10 & 5 & 1 & & & & & & \\
\hline 1 & 6 & 1 & 6 & 15 & 20 & 15 & 6 & 1 & & & & & \\
\hline 2 & 2 & 1 & 4 & 6 & 4 & & & & & & & & \\
\hline 2 & 3 & 1 & 6 & 15 & 20 & 12 & & & & & & & \\
\hline 2 & 4 & 1 & 8 & 28 & 56 & 64 & 32 & & & & & & \\
\hline 2 & 5 & 1 & 10 & 45 & 120 & 200 & 192 & 80 & & & & & \\
\hline 2 & 6 & 1 & 12 & 66 & 220 & 480 & 672 & 544 & 192 & & & & \\
\hline 3 & 3 & 1 & 9 & 36 & 84 & 117 & 81 & & & & & & \\
\hline 3 & 4 & 1 & 12 & 66 & 220 & 477 & 648 & 432 & & & & & \\
\hline 3 & 5 & 1 & 15 & 105 & 455 & 1335 & 2673 & 3375 & 2025 & & & & \\
\hline 3 & 6 & 1 & 18 & 153 & 816 & 3015 & 7938 & 14499 & 16524 & 8748 & & & \\
\hline 4 & 4 & 1 & 16 & 120 & 560 & 1784 & 3936 & 5632 & 4096 & & & & \\
\hline 4 & 5 & 1 & 20 & 190 & 1140 & 4785 & 14544 & 31520 & 44800 & 32000 & & & \\
\hline 4 & 6 & 1 & 24 & 276 & 2024 & 10536 & 40704 & 117376 & 244224 & 331776 & 221184 & & \\
\hline 5 & 5 & 1 & 25 & 300 & 2300 & 12550 & 51030 & 155900 & 347500 & 515625 & 390625 & & \\
\hline 5 & 6 & 1 & 30 & 435 & 4060 & 27255 & 138606 & 544525 & 1641000 & 3645000 & 5400000 & 4050000 & \\
\hline 6 & 6 & 1 & 36 & 630 & 7140 & 58680 & 369792 & 1834992 & 7210080 & 22083840 & 50388480 & 77262336 & 60466176 \\
\hline
\end{tabular}

Fig. 1: Small non-zero values of $f_{m, n, h}$.

The fourth bulleted item is from Kirchhoff's Matrix Tree Theorem (already mentioned).

The following lemma is an important (but basic) consequence of the symmetry of the problem.

Lemma 2 For all $m, n, h \in \mathbb{N}$, we have $f_{m, n, h}=f_{n, m, h}$.

In Figure 1 we list the non-zero values of $f_{m, n, h}$ when $0 \leq m \leq n \leq 6$. These values were generated using a straightforward backtracking algorithm.

\section{Combinatorial equivalence}

In this section, we will describe some combinatorial objects that are equivalent to $h$-edge spanning forests of $K_{m, n}$.

Let $M$ be an $m \times n(0,1)$-matrix. We define a cycle in $M$ to be a set $\left\{e_{1}, e_{2}, \ldots, e_{2 t}\right\}$ of entries of $M$, such that:

- each $e_{i}$ contains the symbol 1 , and

- for $i \in\{1,2, \ldots, t\}$, we have that (a) $e_{2 i-1}$ belongs to the same row as $e_{2 i}$ and (b) $e_{2 i}$ belongs to the same column as $e_{2 i+1}$ (where we take $e_{2 t+1}=e_{1}$ ).

For example, the matrix

\begin{tabular}{|lllll|}
\hline 0 & 1 & 0 & 1 & 1 \\
0 & 0 & 1 & 0 & 1 \\
1 & 0 & 0 & 0 & 1 \\
1 & 1 & 0 & 0 & 0 \\
\hline
\end{tabular}


has a cycle (consisting of the circled 1's), whereas the matrices

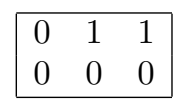
and

\begin{tabular}{|lllll|}
\hline 0 & 1 & 1 & 0 & 1 \\
1 & 0 & 0 & 0 & 1 \\
1 & 0 & 0 & 0 & 0 \\
\hline
\end{tabular}

do not have cycles.

Let $A_{m, n, h}$ be the set of $(0,1)$-matrices with exactly $h$ elements equal to 1 . Let $p_{m, n, h}$ be the probability that an element of $A_{m, n, h}$ chosen uniformly at random contains a cycle. An anonymous user of math . stackexchange.com asked (ii) for a formula for $p_{m, n, h}$. In fact, this was the original motivation for the author to study this problem.

Lemma 3 For all $m, n, h \in \mathbb{N}$, we have $f_{m, n, h}$ is the number of $m \times n(0,1)$-matrices with $h 1$ 's that do not contain a cycle, and hence

$$
f_{m, n, h}=\left(1-p_{m, n, h}\right)\left(\begin{array}{c}
m n \\
h
\end{array}\right)
$$

Proof: A matrix $M \in A_{m, n, h}$ can be interpreted as the biadjacency matrix (iii) of an $h$-edge subgraph $G=G(M)$ of $K_{m, n}$. A cycle in $M \in A_{m, n, h}$ corresponds to a cycle in $G$. Hence

$$
\begin{aligned}
f_{m, n, h} & =\left(1-p_{m, n, h}\right)\left|A_{m, n, h}\right| \\
& =\left(1-p_{m, n, h}\right)\left(\begin{array}{c}
m n \\
h
\end{array}\right) .
\end{aligned}
$$

A problem related to the $(0,1)$-matrix problem above comes from the study of $(0,1)$-matrices that do not have a submatrix which is the incidence matrix of any cycle of length at least 3 ; these are called "totally balanced matrices" (see e.g. [2]).

Another interpretation of matrices in $A_{m, n, h}$ is as induced subgraphs of $K_{m} \square K_{n}$, where $\square$ represents the Cartesian product of graphs. The graph $K_{m} \square K_{n}$ is sometimes called the "rook's graph", since the edges represent the legal moves of a rook on an $m \times n$ chess board. The vertices in $K_{m} \square K_{n}$ are $\{(i, j)$ : $1 \leq i \leq m$ and $1 \leq j \leq n\}$ and there is an edge between distinct vertices $(i, j)$ and $\left(i^{\prime}, j^{\prime}\right)$ whenever $i=i^{\prime}$ or $j=j^{\prime}$. The graph $K_{m} \square K_{n}$ is also the line graph of $K_{m, n}$.

There is a bijection between induced subgraphs $H=H(M)$ of $K_{m} \square K_{n}$ and $(0,1)$-matrices $M=$ $\left(m_{i j}\right) \in A_{m, n, h}$ : we include the vertex $(i, j)$ in $H$ if and only if $m_{i j}=1$. For example, if we ignore the vertices marked 0 in

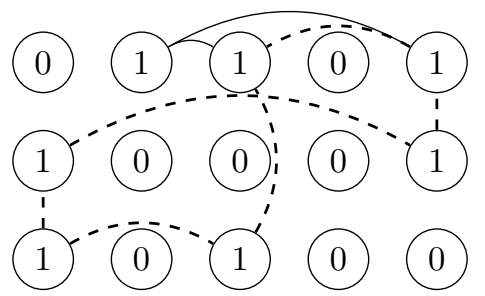

(ii) Full URL: http: //math. stackexchange.com/q/24800

(iii) The biadjacency matrix of a bipartite graph $G$ on the vertex set $\left\{u_{i}\right\}_{1 \leq i \leq m} \cup\left\{v_{j}\right\}_{1 \leq j \leq n}$ is the $m \times n(0,1)$-matrix $M=\left(m_{i j}\right)$ with $m_{i j}=1$ if and only if $u_{i} v_{j}$ is an edge in $G$. 
we obtain an induced subgraph $H(M)$ of $K_{3} \square K_{5}$, corresponding to the matrix

$$
M=\begin{array}{ccccc|}
0 & 1 & 1 & 0 & 1 \\
1 & 0 & 0 & 0 & 1 \\
1 & 0 & 1 & 0 & 0
\end{array}
$$

in $A_{3,5,6}$. We see that cycles in $M$ map from cycles $C_{k}$ in $H$ for some $k \in\{4,6,8, \ldots\}$ (an example of a 6 -cycle is highlighted). There are other cycles in $H$ (e.g. $C_{3}$ in the above example; in fact, if $M$ above had a row of 1's, then $H$ would have a $K_{5}$ subgraph). However, induced cycles $C_{k}$ in $H(M)$ of length $k \in\{4,6,8, \ldots\}$ are in one-to-one correspondence with cycles in $M$ via the above bijection.

Lemma 4 For all $m, n, h \in \mathbb{N}$, we have that $f_{m, n, h}$ is the number of $h$-vertex induced subgraphs of $K_{m} \square K_{n}$ that do not contain an induced $C_{k}$ for any $k \in\{4,6,8, \ldots\}$.

\section{Simplifying the equation}

\subsection{A formula for $f_{m, n, h}$}

We will look at two related ways of simplifying $f_{m, n, h}$. Let $K_{m, n}$ have the vertex bipartition $M \cup N$, where $M=\left\{u_{1}, u_{2}, \ldots, u_{m}\right\}$ and $N=\left\{v_{1}, v_{2}, \ldots, v_{n}\right\}$. Let $B_{m, n, h}$ be the set of $h$-edge spanning forests of $K_{m, n}$. Hence $f_{m, n, h}=\left|B_{m, n, h}\right|$. Define

$$
W_{m, n, h}=\left\{G \in B_{m, n, h}: G \text { has no isolated vertices }\right\}
$$

and $w_{m, n, h}=\left|W_{m, n, h}\right|$. We observe the following:

- Given any spanning forest in $B_{m, n, h}$ we can delete the isolated vertices to obtain a spanning forest in $W_{i, j, h}$ for some $i \leq m$ and $j \leq n$.

- Conversely, given any spanning forest in $W_{i, j, h}$, we can add isolated vertices to it in $\left(\begin{array}{c}m \\ m-i\end{array}\right)\left(\begin{array}{c}n \\ n-j\end{array}\right)$ ways to obtain a spanning forest in $B_{m, n, h}$.

In both of the operations above, we need to relabel the vertices, but we preserve the order of the indices of the non-isolated vertices within $M$ and $N$.

Hence

$$
f_{m, n, h}=\sum_{i=0}^{m} \sum_{j=0}^{n}\left(\begin{array}{c}
m \\
i
\end{array}\right)\left(\begin{array}{l}
n \\
j
\end{array}\right) w_{i, j, h} .
$$

This formula eliminates the need to further account for isolated vertices.

We can decompose any $G \in W_{m, n, h}$ into disjoint components, each of which belong to $W_{i, j, k}$ for some $i \leq m$ and $j \leq n$ and $k \leq h$. Hence any $G \in W_{m, n, h}$ decomposes into the following.

- A partition $P$ of $M$ where $x, y \in M$ belong to the same part if there is a path from $x$ to $y$ in $G$.

- A partition $Q$ of $N$ where $x^{\prime}, y^{\prime} \in N$ belong to the same part if there is a path from $x^{\prime}$ to $y^{\prime}$ in $G$.

- A bijection $\alpha: P \rightarrow Q$ such that every edge $a b$ in $G$ has $a \in p$ and $b \in \alpha(p)$ for some $p \in P$. Hence we have $|P|=|Q|$. 
- For each $p \in P$, a subgraph in $W_{|p|,|\alpha(p)|,|p|+|\alpha(p)|-1}$ induced by the vertices in $p \cup \alpha(p)$.

Regarding the final bulleted item, we observe that the subgraph induced by $p \cup \alpha(p)$ for some $p \in P$ must be a spanning tree on $K_{|p|,|\alpha(p)|}$. Any more edges would cause a cycle, while fewer edges would cause disjoint components (in which case $G$ could be further decomposed).

We give an example of a graph $G$ in $W_{3,5,6}$ below.

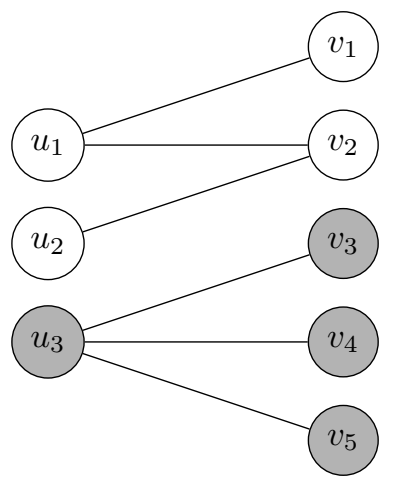

Here, the partitions $P$ and $Q$ are given by $P=\left\{\left\{u_{1}, u_{2}\right\},\left\{u_{3}\right\}\right\}$ and $Q=\left\{\left\{v_{1}, v_{2}\right\},\left\{v_{3}, v_{4}, v_{5}\right\}\right\}$ and we have the bijection $\alpha$ such that

$$
\begin{aligned}
\left\{u_{1}, u_{2}\right\} & \mapsto\left\{v_{1}, v_{2}\right\} \\
\left\{u_{3}\right\} & \mapsto\left\{v_{3}, v_{4}, v_{5}\right\} .
\end{aligned}
$$

We see that $G$ decomposes into a graph in $W_{2,2,3}$ and a graph in $W_{1,3,3}$.

In general, for any $p \in P$, we must have exactly $|p|+|\alpha(p)|-1$ edges in $p \cup \alpha(p)$. Summing this over all $p \in P$ gives $h=m+n-|P|$. We conclude that

$$
|P|=m+n-h
$$

(and thus $|Q|=m+n-h$ ).

We will now describe how to construct any graph in $W_{m, n, h}$ via its decomposition. Let $\mathcal{P}$ be the set of partitions of $M$ and let $\mathcal{Q}$ be the set of partitions of $N$. Given (a) a partition $P \in \mathcal{P}$ of size $|P|=m+n-h$, (b) a partition $Q \in \mathcal{Q}$ and (c) a bijection $\alpha: P \rightarrow Q$, we can construct $\prod_{p \in P} w_{|p|,|\alpha(p)|,|p|+|\alpha(p)|-1}$ distinct graphs $G \in W_{m, n, h}$ by the following steps:

1. Start with $G$ as the graph with vertex set $M \cup N$ and no edges.

2. For each $p \in P$ add one of the subgraphs in $W_{|p|,|\alpha(p)|,|p|+|\alpha(p)|-1}$ on the vertices $p \cup \alpha(p)$.

Since all graphs in $W_{m, n, h}$ can be constructed uniquely by the above steps we have the following theorem.

Theorem 1 For $m, n, h \in \mathbb{N}$,

$$
f_{m, n, h}=\sum_{i=0}^{m} \sum_{j=0}^{n}\left(\begin{array}{c}
m \\
i
\end{array}\right)\left(\begin{array}{c}
n \\
j
\end{array}\right) w_{i, j, h}
$$


where

$$
w_{m, n, h}=\sum_{\substack{P \in \mathcal{P} \\|P|=m+n-h}} \sum_{\substack{Q \in \mathcal{Q} \\|Q|=m+n-h}} \sum_{\substack{\alpha: P \rightarrow Q \\ \text { is a bijection }}} \prod_{p \in P} w_{|p|,|\alpha(p)|,|p|+|\alpha(p)|-1}
$$

where

$$
w_{|p|,|\alpha(p)|,|p|+|\alpha(p)|-1}=|p|^{|\alpha(p)|-1}|\alpha(p)|^{|p|-1} .
$$

\subsection{An improved formula for $f_{m, n, h}$}

We will derive two further equations for $f_{m, n, h}$ (in Theorems 2 and 3), which are related to Theorem 1 . They result in a slightly more complicated algorithm, but will allow us to compute $f_{m, n, h}$ faster than (2).

For all $m, n, h \in \mathbb{N}$, define

$$
W_{m, n, h}^{\prime}=\left\{G \in W_{m, n, h}: \text { the vertices in } N \text { have degree } \geq 2\right\} .
$$

Given any $G \in B_{m, n, h}$ we can delete the isolated vertices and the leaves (vertices of degree 1) in $N$ to obtain a spanning forest in $W_{i, j, h-k}^{\prime}$ for some $i \leq m$ and $j \leq n$ and $k \in \mathbb{N}$. Conversely, given any $G \in W_{i, j, h-k}^{\prime}$, we can add:

- $m-i$ isolated vertices to $M$ in $\left(\begin{array}{c}m \\ m-i\end{array}\right)$ ways, so as to increase $|M|$ to $m$, then

- $n-j$ isolated vertices to $N$ in $\left(\begin{array}{c}n \\ n-j\end{array}\right)$ ways, so as to increase $|N|$ to $n$, then

- $k$ edges to $G$ in $\left(\begin{array}{c}n-j \\ k\end{array}\right) m^{k}$ ways, so as to increase the number of leaves in $N$ by $k$,

thereby obtaining a spanning forest in $B_{m, n, h}$. Similar to the derivation of (2), we need to relabel the vertices so as to preserve the order of the indices of the non-isolated vertices within $M$ and the nonisolated non-leaf vertices within $N$. Hence

$$
f_{m, n, h}=\sum_{i=0}^{m} \sum_{j=0}^{n} \sum_{k=0}^{\min (n-j, h)}\left(\begin{array}{c}
m \\
i
\end{array}\right)\left(\begin{array}{c}
n \\
j, k, n-j-k
\end{array}\right) m^{k} w_{i, j, h-k}^{\prime} .
$$

All of the steps involved for finding the formula (3) for $w_{m, n, h}$ are still valid for $w_{m, n, h}^{\prime}$. Hence (3) remains true if we replace $w$ with $w^{\prime}$. However, we can no longer make use of the simple formula for $w_{|p|,|\alpha(p)|,|p|+|\alpha(p)|-1}$. Nevertheless, we will be able to find a formula for $w_{|p|,|\alpha(p)|,|p|+|\alpha(p)|-1}^{\prime}$ via Inclusion-Exclusion.

For $I \subseteq N$, define

$$
A_{I}=\left\{G \in W_{m, n, m+n-1} \text { : vertices in } I \text { are leaves }\right\} .
$$

Note that spanning forests in $A_{I}$ might also have leaves outside of $I$. Hence $\left|A_{\emptyset}\right|=\left|W_{m, n, m+n-1}\right|=$ $m^{n-1} n^{m-1}$. By symmetry, if $I, J \subseteq N$ and $|I|=|J|$ then $\left|A_{I}\right|=\left|A_{J}\right|$. So we will assume $I=$ $\{n-i+1, n-i+2, \ldots, n\}$. We can construct the graphs in $A_{I}$ by adding $i$ leaves to the graphs in $W_{m, n-|I|, m+n-|I|-1}$ (i.e., the set of spanning trees of $K_{m, n-|I|}$ ), and these leaves can be added in $m^{i}$ ways. Hence

$$
\begin{aligned}
\left|A_{I}\right| & =m^{i} \cdot \text { number of spanning trees of } K_{m, n-i} \\
& =m^{i} m^{n-i-1}(n-i)^{m-1} \\
& =m^{n-1}(n-i)^{m-1}
\end{aligned}
$$


when $m \geq 1$ and $n \geq 1$. Here we take $0^{0}=1$, since we want to account for $K_{1,0} \in W_{1,0,0}$. Hence, by Inclusion-Exclusion, we find

$$
\begin{aligned}
w_{m, n, m+n-1}^{\prime} & =\left|A_{\emptyset}\right|-\left|\bigcup_{\substack{I \subseteq N \\
I \neq \emptyset}} A_{I}\right| \\
& =m^{n-1} n^{m-1}-\sum_{i=1}^{n}(-1)^{i-1}\left(\begin{array}{c}
n \\
i
\end{array}\right) m^{n-1}(n-i)^{m-1} \\
& =m^{n-1} \sum_{i=0}^{n}(-1)^{i}\left(\begin{array}{c}
n \\
i
\end{array}\right)(n-i)^{m-1}
\end{aligned}
$$

when $m \geq 1$ and $n \geq 1$. By definition, we also have $w_{m, n, m+n-1}^{\prime}=0$ when either $m=0$ or $n=0$ (and $w_{m, n, m+n-1}^{\prime}$ is not defined when $m=0$ and $n=0$ ).

Before identifying formulae for $w_{m, n, h}$ and $w_{m, n, h}^{\prime}$ in the next section, we make the following observation.

Lemma 5 For all $m, n, h \in \mathbb{N}$, we have $n$ ! divides $w_{m, n, h}^{\prime}$.

Proof: The symmetric group on $N$ acts on $W_{m, n, h}^{\prime}$ by permuting the vertices in $N$. Suppose a graph $G \in W_{m, n, h}^{\prime}$ has a non-trivial stabiliser subgroup under this action. Then there exists a non-identity permutation $\alpha$ such that $\alpha G=G$, and distinct vertices $v, v^{\prime} \in N$ for which $\alpha(v)=v^{\prime}$. Since $G \in$ $W_{m, n, h}^{\prime}$, the degree of $v$ is 2 or more, so assume $v$ has distinct neighbours $a, b \in M$. Then, since $\alpha G=G$, we find that $v^{\prime}=\alpha(v)$ has the neighbours $a$ and $b$ too. Thus, $\left\{v, v^{\prime}, a, b\right\}$ induces a 4-cycle, giving a contradiction. Hence, all stabiliser subgroups are trivial, and by the Orbit-Stabiliser Theorem, all orbits have size $n$ !. Hence $n$ ! divides $w_{m, n, h}^{\prime}$.

\subsection{Formulae for $w_{m, n, h}$ and $w_{m, n, h}^{\prime}$}

Now we will simplify (3), noting that our simplifications remain valid when we replace $w$ with $w^{\prime}$.

For $a \geq 1$ and $t \geq 1$, let $S_{a, t}$ be the set of (number) partitions of $a$ into $t$ non-zero parts. We will interpret elements of $S_{a, t}$ as multisets; for example $\{4,3,3,1,1\} \in S_{12,5}$. We will require $S_{a, t}=\emptyset$ when $t<0$, and $S_{0,0}=\{\emptyset\}$ and $S_{0, t}=\emptyset$ when $t>0$. For $z \in S_{a, t}$, let $T[z]$ be the set of partitions of $\{1,2, \ldots, a\}$ whose part sizes induce the number partition $z$. For $z \in S_{a, t}$, let $\hat{z}$ denote an arbitrary element of $T[z]$. If $P \in T[x]$ and $Q \in T[z]$, then any bijection $\alpha: P \rightarrow Q$ induces a bijection $\beta: x \rightarrow z$. 
Hence

$$
\begin{aligned}
& w_{m, n, h}=\sum_{x, z \in S_{m, m+n-h}} \sum_{P \in T[x]} \sum_{Q \in T[z]} \sum_{\substack{\alpha: P \rightarrow Q \\
\alpha \text { is a bijection }}} \prod_{p \in P} w_{|p|,|\alpha(p)|,|p|+|\alpha(p)|-1} \\
& =\sum_{x, z \in S_{m, m+n-h}} \sum_{P \in T[x]} \sum_{Q \in T[z]} \sum_{\substack{\alpha: \hat{x} \rightarrow \hat{z} \\
\alpha \text { is a bijection }}} \prod_{p \in \hat{x}} w_{|p|,|\alpha(p)|,|p|+|\alpha(p)|-1} \\
& =\sum_{x, z \in S_{m, m+n-h}}|T[x]| \cdot|T[z]| \sum_{\substack{\alpha: \hat{x} \rightarrow \hat{z} \\
\alpha \text { is a bijection }}} \prod_{p \in \hat{x}} w_{|p|,|\alpha(p)|,|p|+|\alpha(p)|-1} \\
& =\sum_{x, z \in S_{m, m+n-h}}|T[x]| \cdot|T[z]| \sum_{\substack{\beta: x \rightarrow z \\
\beta \text { is a bijection }}} \prod_{r \in x} w_{r, \beta(r), r+\beta(r)-1} .
\end{aligned}
$$

If $z \in S_{a, t}$, then

$$
|T[z]|=\frac{a !}{\prod_{i \geq 1} i !^{s_{i}(z)} s_{i}(z) !}
$$

where $s_{i}(z)$ denotes the number of parts $i$ in $z$ (see e.g. [1, Theorem 13.2]). Hence we have the following theorems.

Theorem 2 For $m, n, h \in \mathbb{N}$,

$$
f_{m, n, h}=\sum_{i=0}^{m} \sum_{j=0}^{n}\left(\begin{array}{c}
m \\
i
\end{array}\right)\left(\begin{array}{c}
n \\
j
\end{array}\right) w_{i, j, h}
$$

where

$$
w_{m, n, h}=\sum_{x, z \in S_{m, m+n-h}} \sum_{\substack{\beta: x \rightarrow z \\ \beta \text { is a bijection }}} \frac{m !}{\prod_{i \geq 1} i !^{s_{i}(x)} s_{i}(x) !} \frac{n !}{\prod_{i \geq 1} i !^{s_{i}(z)} s_{i}(z) !} \prod_{r \in x} r^{\beta(r)-1} \beta(r)^{r-1} .
$$

Theorem 3 For $m, n, h \in \mathbb{N}$,

$$
f_{m, n, h}=\sum_{i=0}^{m} \sum_{j=0}^{n} \sum_{k=0}^{\min (n-j, h)}\left(\begin{array}{c}
m \\
i
\end{array}\right)\left(\begin{array}{c}
n \\
j, k, n-j-k
\end{array}\right) m^{k} w_{i, j, h-k}^{\prime}
$$

where

$$
w_{m, n, h}^{\prime}=\sum_{x, z \in S_{m, m+n-h}} \sum_{\substack{\beta: x \rightarrow z \\ \beta \text { is a bijection }}} \frac{m !}{\prod_{i \geq 1} i !^{!_{i}(x)} s_{i}(x) !} \frac{n !}{\prod_{i \geq 1} i !^{s_{i}(z)} s_{i}(z) !} \prod_{r \in x} w_{r, \beta(r), r+\beta(r)-1}^{\prime}
$$

where

$$
w_{m, n, m+n-1}^{\prime}=m^{n-1} \sum_{i=0}^{n}(-1)^{i}\left(\begin{array}{c}
n \\
i
\end{array}\right)(n-i)^{m-1} .
$$

The main advantage of Theorems 2 and 3 over Theorem 1 is the summation is over number partitions (rather than set partitions). 


\section{Implementation}

The author has implemented the two formulae for $f_{m, n, h}$ described in the preceding section, along with a simple backtracking algorithm. The results between all three implementations concur, giving confidence in the accuracy of the code. The $\mathrm{C}$ source code is available as supplementary material to this document. The GMP library was used for arbitrary precision arithmetic [6].

The formula involving $w_{m, n, h}^{\prime}$ instead of $w_{m, n, h}$ was (unsurprisingly) much faster, largely because many values of $w_{m, n, h}^{\prime}$ equal 0 , such as when $m \leq n$, which can be used to drastically reduce the search tree.

\subsection{Symmetry breaking}

The run-time of the program was also improved through the use of symmetry breaking, which we will now describe. For $x \in S_{m, m+n-h}$ and $z \in S_{n, m+n-h}$, there are often many bijections $\beta: x \rightarrow z$ which map the same elements to the same elements (since both $x$ and $z$ are multisets). In these instances, a naïve algorithm would repeat the same computation unnecessarily. Let $x$ and $z$ be the multisets $x=$ $\left\{x_{1}, x_{2}, \ldots, x_{m+n-h}\right\}$ and $z=\left\{z_{1}, z_{2}, \ldots, z_{m+n-h}\right\}$. We define the condition:

Symmetry breaking condition 1: We say $\beta: x \rightarrow z$ is half-canonical if $\beta^{-1}\left(z_{i-1}\right)<\beta^{-1}\left(z_{i}\right)$

whenever $z_{i-1}=z_{i}$.

We need to add a multiplicative factor to adjust for the restriction to half-canonical bijections. Hence

$w_{m, n, h}=\sum_{x, z \in S_{m, m+n-h}} \sum_{\substack{\beta: x \rightarrow z \\ \beta \text { is a abjection } \\ \beta \text { is half-canonical }}}\left(\prod_{i \geq 1} i !^{s_{i}(z)}\right) \frac{m !}{\prod_{i \geq 1} i !^{s_{i}(x)} s_{i}(x) !} \frac{n !}{\prod_{i \geq 1} i !^{s_{i}(z)} s_{i}(z) !} \prod_{r \in x} w_{r, \beta(r), r+\beta(r)-1}$

and similarly with $w^{\prime}$ in place of $w$. Using this assumption, we reduce the number of bijections by a factor of $\prod_{i \geq 1} i !^{s_{i}(z)}$, which results in a substantial time saving.

Instead of the single symmetry breaking assumption, it is possible to utilise symmetry breaking using an additional condition:

Symmetry breaking condition 2: We say $\beta: x \rightarrow z$ is canonical if it is half-canonical and $\beta\left(x_{i-1}\right)<\beta\left(x_{i}\right)$ whenever $x_{i}=x_{i-1}$.

Again, we find

$$
w_{m, n, h}=\sum_{x, z \in S_{m, m+n-h}} \sum_{\substack{\beta: x \rightarrow z \\ \beta \text { is a bijection } \\ \beta \text { is canonical }}} \Gamma_{x, z, \beta} \frac{m !}{\prod_{i \geq 1} i !^{s_{i}(x)} s_{i}(x) !} \frac{n !}{\prod_{i \geq 1} i l^{s_{i}(z)} s_{i}(z) !} \prod_{r \in x} w_{r, \beta(r), r+\beta(r)-1},
$$

and similarly with $w^{\prime}$ in place of $w$, where $\Gamma_{x, z, \beta}$ is the number of bijections between the multisets $x$ and $z$ which map the same elements to the same elements (as $\beta$ ). A formula for $\Gamma_{x, z, \beta}$ was given by e.g. [3], namely

$$
\Gamma_{x, z, \beta}=\frac{\prod_{i \geq 1} s_{i}(x) ! \prod_{i \geq 1} s_{i}(z) !}{\prod_{i \geq 1} \prod_{j \geq 1} s_{i, j}(x, \beta) !}
$$

where 
- $s_{i}(z)$ denotes the number of parts $i$ in $z$ (as before), and

- $s_{i, j}(x, \beta)$ is the number of elements $(i, j)$ in the multiset $\{(r, \beta(r)): r \in x\}$.

The author has implemented both of these symmetry breaking schemes in order to compare their performance (see Section 5.3).

\subsection{Pseudo-code}

Algorithm 1 gives a pseudo-code version of the $\mathrm{C}$ code used to implement the algorithm described by Theorem 3 using the half-canonical symmetry breaking condition. The partitions of $m$ and $n$ into $k$ parts were computed whenever needed and stored in memory. Iterating through the half-canonical bijections was performed "on the fly" using a backtracking algorithm.

While Theorems 2 and 3 are valid for all $m, n, h \in \mathbb{N}$, we need to set $f_{m, n, 0}=1$ separately in the $\mathrm{C}$ code.

\subsection{Performance}

The $\mathrm{C}$ code was run on a $2 \times 2.66 \mathrm{GHz}$ processor (although the code itself is not parallelised). The following table gives the run-times (in seconds) for the two algorithms under the two symmetry breaking schemes when computing all non-zero $f_{m, n, h}$ with $m, n \leq 19$.

\begin{tabular}{l|rr} 
& half-canonical & canonical \\
\hline Theorem[2] & 27.1 & 6.8 \\
Theorem 3 & 4.1 & 4.5
\end{tabular}

The fastest version is Theorem 3 using half-canonical symmetry breaking. Under these conditions, the code had the following run-times to find all non-zero values of $f_{m, n, h}$ with $m, n \leq t$ :

\begin{tabular}{r|r}
$t$ & time (sec) \\
\hline 25 & 20.5 \\
26 & 27.7 \\
27 & 35.4 \\
28 & 46.0 \\
29 & 62.2 \\
30 & 83.0
\end{tabular}

This table indicates the scalability of the program, which is not overwhelming for these values of $t$. The author also ran the program to compute $f_{m, n, h}$ with $m, n \leq t$ where $t=50$, which took under 2 days (to be precise, it took 1 day 15 hours and 17 minutes). The largest number encountered was $f_{50,50,101}$, which has 167 digits, and is equal to the number of spanning trees of $K_{50,50}$, which is $50^{50-1} \cdot 50^{50-1}=50^{98}$.

\subsection{Complexity}

In the worst case, $x=z=\{1,2,3, \ldots, t\}$, where $\min (m, n)=1+2+\cdots+t=\frac{1}{2} t(t+1)$ (so $t=O(\sqrt{\min (m, n)})$, in which case the program must iterate through all $t$ ! bijections from $x$ to $z$. So, for a worst case analysis, we assume $m=n$ and $m+n-h=\lfloor\sqrt{m}\rfloor$. 


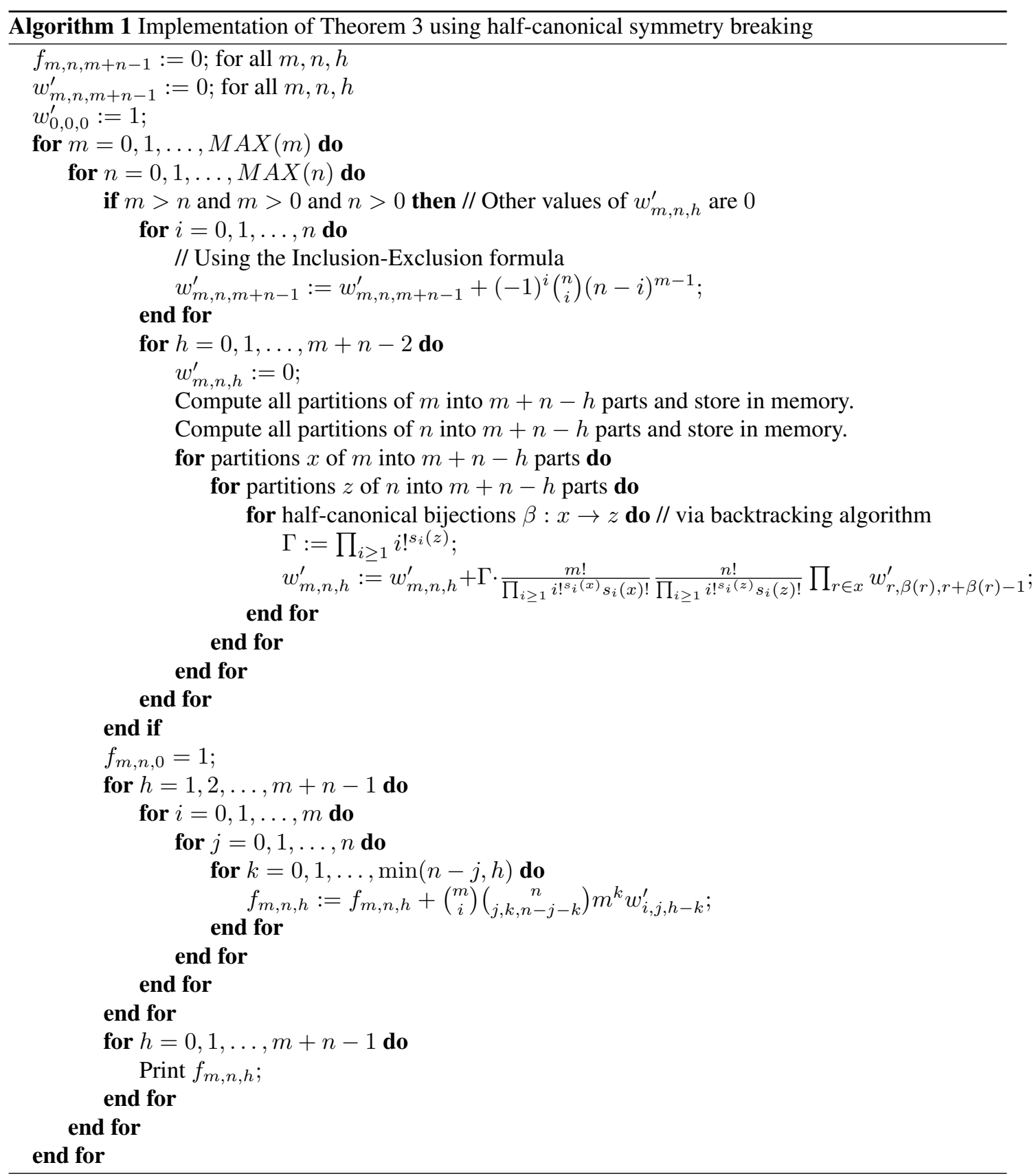


Hence, given $x, z \in S_{m, m+n-h}$, there can be $O(\lfloor\sqrt{m}\rfloor !)$ canonical bijections from $x$ to $z$. Hardy and Ramanujan's asymptotic formula for the number of partitions of $m$, namely

$$
\frac{1}{4 m \sqrt{3}} e^{\pi \sqrt{2 m / 3}}
$$

gives a crude asymptotic upper bound on $\left|S_{m,\lfloor\sqrt{m}\rfloor}\right|^{2}$, specifically

$$
\left|S_{m,\lfloor\sqrt{m}\rfloor}\right|^{2}=O\left(\frac{e^{\text {const } m}}{m^{2}}\right)
$$

as the number of ways of choosing $x$ and $z$. Hence, 4 has

$$
O\left(e^{\text {const } \sqrt{m}} m^{\sqrt{m} / 2+\text { const }}\right)
$$

terms, by Stirling's Approximation. In contrast, a backtracking algorithm would need to generate and check around $\left(\begin{array}{c}m^{2} \\ \lfloor\sqrt{m}\rfloor\end{array}\right) \leq \frac{1}{\lfloor\sqrt{m}\rfloor !} m^{2\lfloor\sqrt{m}\rfloor}$ graphs, which, when $m=n$ and $h=2 m-\sqrt{m}$, is

$$
O\left(e^{\sqrt{m}} m^{1.5 \sqrt{m}+\text { const }}\right)
$$

iterations, by Stirling's Approximation. Of course, when implementing these algorithms, we use pruning whenever possible to reduce the search space, which makes a drastic difference not accounted for in these approximations.

\subsubsection{When $h$ is fixed}

We conclude this paper with the observation that, when $h$ is fixed, computing $f_{m, n, h}$ is asymptotically "easy". The underlying reason is that, for sufficiently large $m$ or $n$, we must have isolated vertices in graphs in $B_{m, n, h}$. Thus, (2) only contains a finite number of non-zero terms.

Theorem 4 For fixed $h$, computing $f_{m, n, h}$ can be performed in time $O(\log (m n))$.

Proof: If $i>h$ or $j>h$, then $w_{i, j, h}=0$ (since any graph in $B_{i, j, h}$ must have an isolated vertex). Hence, (2) is equivalent to

$$
f_{m, n, h}=\sum_{i=0}^{h} \sum_{j=0}^{h}\left(\begin{array}{c}
m \\
i
\end{array}\right)\left(\begin{array}{c}
n \\
j
\end{array}\right) w_{i, j, h} .
$$

For fixed $h$, there is a finite number of terms in this sum. Thus, for fixed $h$, we could write a program in which:

- we store a list of the pairs $(i, j)$ for which $w_{i, j, h}$ is non-zero, along with the value of $w_{i, j, h}$,

- we iterate through this list, computing $\left(\begin{array}{c}m \\ i\end{array}\right)\left(\begin{array}{c}n \\ j\end{array}\right) w_{i, j, h}$, and add it to a running total.

We can compute $\left(\begin{array}{c}m \\ i\end{array}\right)=\frac{1}{i !} m(m-1) \cdots(m-i+1)$ using $O(h)$ multiplications (since $i \leq h$ ), each of which takes time $O(\log m)$, and one division. Hence, $\left(\begin{array}{c}m \\ i\end{array}\right)$ can be computed in time $O(\log m)$ time (since $h$ is fixed). Similarly $\left(\begin{array}{l}n \\ j\end{array}\right)$ can be computed in $O(\log n)$ time. We conclude that the whole summation can be performed in time $O(\log (m n))$. 


\section{Acknowledgements}

The author would like to thank Graham Farr for his feedback. The author would also like to thank Zur

Luria for directing her to [3]. Thanks also to mathoverflow. net and math. stackexchange. com for providing a forum to discuss problems arising in this work.

\section{References}

[1] G. E. ANDREws, The theory of partitions, Cambridge University Press, 1984.

[2] R. P. Anstee And M. FARBer, Characterizations of totally balanced matrices, J. Algorithms, 5 (1984), pp. 215-230.

[3] A. BARVINOK, Enumerating contingency tables via random permanents, Combin. Probab. Comput., 17 (2008), pp. 1-19.

[4] A. BuöKlund, T. Husfeldt, P. KAski, And M. Koivisto, Computing the Tutte polynomial in vertex-exponential time, in Proc. of the 49th Annual IEEE Symposium on Foundations of Computer Science (FOCS 2008), 2008, pp. 677-686.

[5] G. FARR AND C. MCDIARMID, The complexity of counting homeomorphs, Theoretical Computer Science, 36 (1985), pp. 345-348.

[6] T. GRANLUND ET AL., GNU multiple precision arithmetic library. http: //gmplib.org/.

[7] Y. Jin AND C. LIU, The enumeration of labelled spanning trees of $K_{m, n}$, Australas. J. Combin., 28 (2003), pp. 73-79.

[8] — Enumeration for spanning forests of complete bipartite graphs, Ars Combin., 70 (2004), pp. $135-138$.

[9] C. J. LiU AND Y. CHOw, Enumeration of forests in a graph, Proc. Amer. Math. Soc., 83 (1981), pp. 659-662.

[10] W. MYRvold, Counting k-component forests of a graph, Networks, 22 (1992), pp. 647-652.

[11] T. Porter, Generating the list of spanning trees in $K_{s, t}$, J. Combin. Math. Combin. Comput., 50 (2004), pp. 17-32.

[12] L. A. SzÉKELY, Counting rooted spanning forests in complete multipartite graphs, Ars Combin., 73 (2004).

[13] Y. TERANISHI, The number of spanning forests of a graph, Discrete Math., 290 (2005), pp. $259-267$. 